\title{
The effect of finite size of the system on correlation length behaviour at the presence of external field
}

\author{
M.P.Kozlovskii \\ Institute for Condensed Matter Physics of the National Academy of Sciences of Ukraine, \\ 1 Svientsitskii Str., 79011 Lviv, Ukraine
}

Received May 4, 2007, in final form May 31, 2007

The explicit analytical expression for the effective correlation length $\xi$ as a function of reduced temperature $\tau$, external field $h$ and system size $L$ for the Ising-like system near the phase transition point $T_{\mathrm{c}}$ is obtained. The role of these quantities in the formation of the correlation length value is ascertained. It is shown that the irregular increase of the correlation length exists only in the case of $\tau \rightarrow 0, h \rightarrow 0$ and $L \rightarrow \infty$. With deviation from these values the essential slowing down exists for the increasing $\xi$. The criterium of the permissible range of temperature values (field values), where the correlation length behaviour is defined by temperature (or field) variable, is established for the fixed size of the system. Beyond this range $\tau<\tau_{\mathrm{c}}, h<h_{\mathrm{cr}}$ the system size becomes crucial for forming the correlation length.

Key words: phase transition, correlation length, external field, finite size system

PACS: $05.50 .+q, 64.60 . F r, 75.10 . H k$

The correlation length $\xi$ is one of the most important characteristics of the phase transition. This quantity tends to infinity with nearing to the phase transition temperature $T_{\mathrm{c}}$ from exponential law

$$
\xi=\xi_{0 t}|\tau|^{-\nu},
$$

at the absence of the external field for infinite systems $\left(\tau=\left(T-T_{\mathrm{c}}\right) / T_{\mathrm{c}}\right)$. The quantity $\xi_{0 t}$ is called critical amplitude, and $\nu$ is (temperature) critical exponent. Now it is well established that $\nu$ depends on the system universality class (see, for example, $[1,2]$ ) and dimensionality. For Ising model, which is considered below, this value also depends on the spin component dilution (replacing magnetic atoms by nonmagnetic ones) and on the type of the impurity distribution [3,4]. In contrast to the $\nu$ the quantity $\xi_{0 t}$ is nonuniversal and depends on the microscopic parameters of the system. Far from the phase transition point, the quantity $\xi_{0 t}$ takes on the values of the order of the distance between the system particles. The value of the exponent $\nu$ as well as other critical exponents for Ising model are known with high accuracy (see, for example, [5]). These are the so-called temperature critical exponents. In addition, the field exponents that describe the dependence of the physical quantities on the field in the case of $T=T_{\mathrm{c}}$, are known as well. Since for the correlation length $\xi$ we have the dependence

$$
\xi=\xi_{0 h} h^{-\mu},
$$

where $\mu$ is the (filed) critical exponent of the correlation length at $T=T_{\mathrm{c}}, \xi_{0 h}$ is the corresponding critical amplitude, $h=\beta \mathcal{H}\left(\mathcal{H}\right.$ is magnetic field $\left.\beta^{-1}=k T\right)$.

Generalizing different calculation methods ( $\epsilon$ - series expansion [6], theoretical field approach for fixed dimensionality $d=3$ [7], Monte-Carlo calculation data, [8], high-temperature expansions $[9]$ ), one may claim that for Ising model $\nu=0.630$, and $\mu$ is determined using the relations for critical exponents

$$
\mu=\frac{\nu}{\beta+\gamma} .
$$

Taking into account the results of papers [6-9], we find

$$
\mu=0.402 \text {. }
$$


In the recent work [10] the expression for some typical distance $\xi_{e}$ is found, which is proportional to the correlation length of the three-dimensional Ising model. The quantity $\xi_{e}$ explicitly takes into account the dependence on the temperature $\tau$ as well as on the external field $h$ :

$$
\xi_{e}=\xi_{0}\left(\tilde{h}^{2}+\tilde{\tau}^{(d+2) \nu}\right)^{-\frac{1}{d+2}} .
$$

Here $\xi_{0}=c \cdot s_{0}$, where $c$ is the constant of the simple cubic lattice, for which the calculations were performed, and $s_{0}$ is some interaction potential parameter $\left(s_{0}=2\right)$ [11]. The symbols $\tilde{h}$ and $\tilde{\tau}$ are renormalized quantities of the external field $h$ and reduced temperature $\tau$

$$
\tilde{\tau}=\frac{c_{1 k}}{f_{0}} \tau, \quad \tilde{h}=s_{0}^{3 / 2} f_{0}^{-1} h
$$

The values for the coefficients $c_{1 k}$ and $f_{0}$ are given in $[11,12]$. For the Ising systems with exponentially decreasing interaction potential $\Phi\left(r_{i j}\right)=A \exp \left(-r_{i j} / b\right)$ these coefficients take on the values $c_{1 k}=0.976, f_{0}=1 / 2$ at $b / c=0.5$. For other values of Hamiltonian parameter the values for these quantities are given in [13].

As one can see from (4), at sufficiently large fields $\tilde{h} \gg h_{\mathrm{c}}\left(h_{\mathrm{c}}=|\tilde{\tau}|^{p_{0}}, p_{0}=\delta \beta\right.$, where $\delta$ and $\beta$ are critical exponents of the order parameter) the temperature practically does not effect the values of the correlation length. In this case the formula (4) transforms into the dependence (2) with critical amplitude

$$
\xi_{\text {eh }}=\xi_{0} f_{0}^{\mu} s_{0}^{-3 / 5}
$$

and critical exponent

$$
\mu=\frac{2}{d+2} .
$$

In the case of the weak fields $\left(\tilde{h} \ll h_{\mathrm{c}}\right)$ the temperature variable plays a crucial role in the formation of the correlation length. Here the dependence (1) is valid, where for the critical amplitude we have

$$
\xi_{\text {et }}=\xi_{0}\left(\frac{f_{0}}{c_{1 k}}\right)^{\nu} .
$$

The critical exponent $\nu$ in contrast to $\mu$ from (7) is determined by analyzing the recurrence relations [11] for coefficients of the effective block Hamiltonians. It depends on the symmetry of the system, on the presence of impurities, etc. [4].

The expression (4) is the consequence of the temperature and field dependence of the system exit point $n_{p}$ on the order parameter critical fluctuations [10].

$$
n_{p}=-\frac{\ln \left(\tilde{h}^{2}+h_{\mathrm{c}}^{2}\right)}{2 \ln E_{1}}-1,
$$

where $E_{1}$ is the eigenvalue of the linearized matrix of renormalization group transformation (near the fixed point) [11-14].

$$
E_{1}=s^{\frac{d+2}{2}} .
$$

Here $s$ is the renormalization group parameter. The quantity $n_{p}$ was introduced in [13]. It characterizes the number of enlargements of the lattice system of the Ising spins (with parameter $s$ ) in the spirit of the Kadanoff construction [15]. For the fixed temperature $\tau(\tau \ll 1)$ one can construct the sequence of $n_{p}$ effective block lattices, each of which exhibits an $s$ times enlarged lattice constant. In the case of $h=0$ the quantity $n_{p} \rightarrow \infty$, when $\tau \rightarrow 0$. For $\tau \neq 0$ the quantity $n_{p}$ is finite and proportional to $-\ln \tau$ [13].

For the finite systems, which are investigated using the numerical methods $[8,16]$, the system consists of the finite number of particles $L^{3}$. It is evident that the correlation length in such systems does not exceed the value of $L$. This fact essentially effects the way of its calculation. In order to investigate the effect of the system finiteness on the formation of the correlation length we modify the quantity $n_{p}$. It defines the number of the effective block lattices (Kadanoff construction [15]), 
for which the renormalization group symmetry exists. The typical distance $L_{p}=s^{n_{p}}$ corresponds to the quantity $n_{p}$. It is obvious that $\xi \leqslant c L_{p}$.

Let us assume that $T=T_{\mathrm{c}}$ and $h=0$. Then the maximal value of the quantity $n_{p}$ defines the effective block lattice period $c_{n_{p}}$, which is determined only by the system size.

$$
c_{n_{p}}=c_{0} L_{p} .
$$

For the system with $N$ particles $\left(N=L^{3}\right)$ the effective block lattice period $c_{n_{p}}$ cannot exceed the value $L$. Thus, the largest value of $n_{p}$ is defined by the equality

$$
c_{n_{p}}=L \text {. }
$$

Taking into account (11), we find

$$
n_{p}=\frac{1}{3} \frac{\ln N_{0}}{\ln s},
$$

where $N_{0}=N s_{0}^{-3}$. The parameter $s_{0}$ defines the region of the parabolic approximation for the Fourier transform of the interaction potential $[10,13]$. The formula (13) allows one to generalize the expression (9) for the case of the finite systems when $\tau \neq 0$ and $h \neq 0$. We obtain the relation

$$
n_{p}=-\frac{\ln \left(\tilde{h}^{2}+h_{\mathrm{c}}^{2}+L_{0}^{-(d+2)}\right)}{2 \ln E_{1}}-1 .
$$

The value of $n_{p}$ determines the characteristic distance $\xi$ (hereinafter we call it correlation distance) ${ }^{1}$

$$
\xi=\xi_{0} s^{n_{p}},
$$

where $\xi_{0}=c_{0}=c s_{0}$. Using the formula (14) for the quantity $n_{p}$, we find

$$
\xi=\xi_{0}^{\prime}\left(\tilde{h}^{2}+\tilde{\tau}^{(d+2) \nu}+L_{0}^{-(d+2)}\right)^{-\frac{1}{d+2}},
$$

where for the quantity $\xi_{0}^{\prime}$ we have

$$
\xi_{0}^{\prime}=\xi_{0} s^{-1}=c \frac{s_{0}}{s} .
$$

In the limit case $h=0$ and $L \rightarrow \infty$ the expression (16) transforms into (1), where for the critical amplitude $\xi_{0 t}$ we have the relation

$$
\xi_{0 t}=c \frac{s_{0}}{s}\left(\frac{f_{0}}{c_{1 k}}\right)^{\nu} .
$$

In the case of $\tau=0$ and $L \rightarrow \infty$ the expression (16) is reduced to the dependence (2), where for $\xi_{0 h}$ we obtain

$$
\xi_{0 h}=\frac{c}{s}\left(s_{0} f_{0}\right)^{\mu} .
$$

When $\tau=0, h=0$ and $L_{0}=$ const for the quantity $\xi$ we have

$$
\xi_{L}=\xi_{0}^{\prime} L_{0}=\frac{c}{s} L,
$$

where $L$ is the linear size of the cube of the investigated system, $c$ is lattice constant.

From (16) one can estimate the size of the system, which exhibits critical phenomena. It is well known that for the infinite system at $h=0$ such phenomena take place at $\tau<\tau_{\mathrm{c}}$, where $\tau_{\mathrm{c}} \approx 10^{-2}$. The quantity (16) for $\tau=\tau_{\mathrm{c}}$ (at $h=0$ and $L \rightarrow \infty$ ) takes on the value

$$
\xi\left(\tau_{\mathrm{c}}, h=0, L \rightarrow \infty\right) \approx 6 c .
$$

\footnotetext{
${ }^{1}$ Note that in the work [10] the value $\xi$ was defined as $\xi^{\prime}=\xi_{0} s^{n_{p}+1}$, i.e., it was $s$ times larger. It does not effect the asymptotic behaviour in both cases, but changes the numerical value of the critical amplitude.
} 


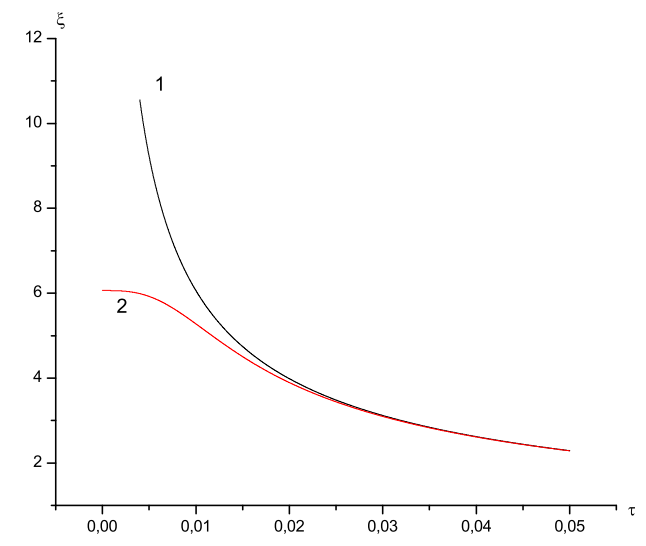

Figure 1. The correlation distance $\xi_{e}$ at $h=0$ (curve 1) and $h=h_{\mathrm{cr}}$ (curve 2) as a function of the temperature.

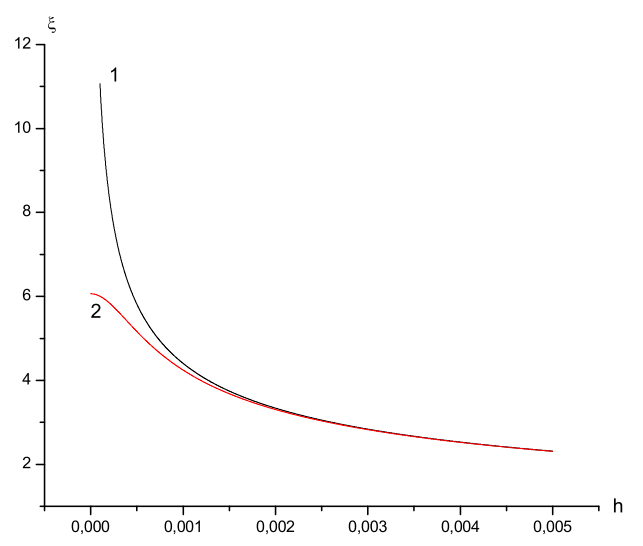

Figure 2. The correlation distance $\xi_{e}$ at $\tau=0$ (curve 1 ) and $\tau=\tau_{\mathrm{c}}$ (curve 2) as a function of the magnetic field.

Let us estimate the value of the field $h$, which defines the quantity (21) at $\tau=0$ and $L \rightarrow \infty$. We have

$$
h=4.5 \cdot 10^{-4} \text {. }
$$

Now we find the value of $L$ corresponding to the case of $\xi=6$ on condition that $\tau=0, h=0$. Using the relation (16) and the equality $\xi=6 c$, we find

$$
L_{\mathrm{c}} \approx 20,
$$

which corresponds to $N \sim 10^{4}$ particles.

Thus the formula (16) allows one to establish the boundaries of the critical region, i.e., the values of the quantities $\tau_{\mathrm{c}}, h_{\mathrm{cr}}$ and the particle number $N_{\mathrm{c}}$ in the system $\left(\tau_{\mathrm{c}}=10^{-2}, h_{\mathrm{cr}} \approx 4.5 \cdot 10^{-4}\right.$ and $N_{\mathrm{c}} \approx 10^{4}$ ), for which the value of the correlation distance is the same on condition that the rest of parameters take on the values corresponding to the critical point.

Let us consider the behavior of the quantity (16) at $\tau<\tau_{\mathrm{c}}, h<h_{\mathrm{cr}}$ and $L>L_{\mathrm{c}}$. For the infinite systems $(L \rightarrow \infty)$ we have only the dependencies on the $\tau$ and $h$. In the cases of $h=0$ and $h=h_{\mathrm{cr}}$ the dependence of $\xi$ on the temperature is shown in figure 1. The dependence of this quantity on the field is shown in figure 2 for the cases of $\tau=0$ and $\tau=0.01$. By comparing the dependencies for $\xi$ on the temperature (figure 1) it is easy to see that at $h=0$ the increase of the correlation distance with a decreasing $\tau$ is permanent and tends to infinity at $\tau \rightarrow 0$. In the case of $h=h_{\text {cr }}$ the increase of $\xi$ with a decreasing $\tau$ tends only to the value of $\xi$ from (21). A similar situation takes place for the dependence of the correlation distance on the field. Note that the dependences shown in figure 1 (at $h=0$ ) and in figure 2 (at $\tau=0$ ) are in good agreement with the corresponding numerical calculations performed using Monte-Carlo method [16].

We investigate the effect of the finite size of the system (with linear size $L$ ) on the quantity $\xi$. At $\tau=0$ and $h=0$ the expression (20) is valid. The quantity $\xi_{L}$ increases linearly with the increase of the quantity $L$.

Let us assume that $h=0$, and $\tau \neq 0$. Then, the dependence $\xi$ on the $L$ has the form shown in figure 3. The presence of a horizontal section of the curve argues about the sufficiently large size of the system, which does not effect the values of the quantity $\xi$. In this case $\xi$ is defined by the temperature and by the field. The sloping part of the curve demonstrates that the quantity $\xi$ is formed only by the quantity $L$, i.e., by the system size.

Thus, at the absence of the field, the behaviour of $\xi$ from (16) in the region of the temperatures $\tau<\tau_{\mathrm{c}}$ can be described only for the case when the linear sizes exceed $L_{\mathrm{c}}$. For temperatures $\tau<\tau_{\mathrm{c}}$ 


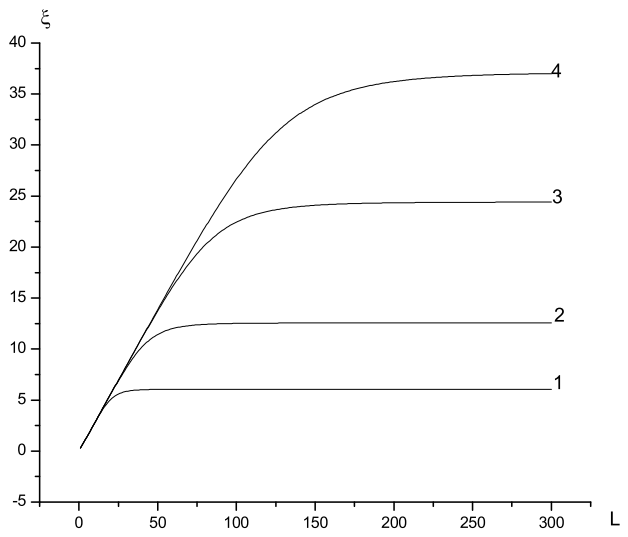

Figure 3. The correlation distance $\xi_{e}$ at $\tau=10^{-2}$ - curve $1, \tau=3 \cdot 10^{-3}$ - curve $2, \tau=10^{-3}$ - curve 3 and $\tau=0,5 \cdot 10^{-3}$ - curve 4 as a function of the values of $L$ (the number of lattice points per direction).

we have $L>L_{c 1} \approx 30$; for $\tau<3 \cdot 10^{-3}$ at $L>L_{c 2} \approx 50$; for $\tau<10^{-3}$ at $L>L_{c 3} \approx 120$ and for $\tau<0,5 \cdot 10^{-3}$ at $L>L_{c 4} \approx 200$ (see figure 3 ). The last value of $L$ corresponds to the particles number $8 \cdot 10^{6}$. With a decreasing region of temperature values we have an essentially larger number of particles which causes numerous problems in performing the numerical calculations.

In the case of $\tau=0$ one can construct the dependence $\xi$ on the system size $L$ for different values of $h<h_{\mathrm{cr}}$. Its character is similar to the one shown in figure 3 .

The general condition for defining the size of the system $L$ at fixed values of temperature $\tau$ and field $h$ is inequality $L>L^{*}$, where

$$
L^{*}=s_{0}\left(\tilde{h}^{2}+\tilde{\tau}^{(d+2) \nu}\right)^{-\frac{1}{d+2}} .
$$

In the region of small values of the field (see [17]) $\tilde{h} \ll h_{\mathrm{c}}$ for $L^{*}$ we have

$$
\mathrm{E}_{\mathrm{c}}^{*}=s_{0} \tilde{\tau}^{-\nu}\left(1-\frac{1}{5} \frac{\tilde{h}^{2}}{h_{\mathrm{c}}^{2}}\right),
$$

and for large values of the field $\tilde{h} \gg h_{\mathrm{c}}$ we find

$$
L_{h}^{*}=s_{0} \tilde{h}^{-2 / 5}\left(1-\frac{1}{5} \frac{h_{\mathrm{c}}^{2}}{\tilde{h}^{2}}\right) .
$$

In the vicinity of pseudocritical line $\left(\tilde{h} \approx h_{\mathrm{c}}\right)$, the quantity $L^{*}$ is defined by the values of temperature and field according to the expression (24).

\section{Conclusions}

The investigations of critical phenomena at the second order phase transition for finite size systems or the use of numerical methods such as Monte-Carlo method, should predict the correlation among particles number, the range of the temperature region and external field values. For the one-component three-dimensional spin system the values of the temperature $\tau_{\mathrm{c}}$ and field $h_{\mathrm{cr}}$ are established which correspond values to the boundary of the critical region. In other words, the critical phenomena take place only in the regions $0<\tau<\tau_{\mathrm{c}}$ and $h<h_{\mathrm{cr}}$, where $\tau_{\mathrm{c}}=10^{-2}$, and 
$h_{\mathrm{cr}}=4.5 \cdot 10^{-4}$. In the case of $h>h_{\mathrm{cr}}$ and $\tau>\tau_{\mathrm{c}}$ the correlation distance becomes too small to observe the critical phenomena.

It is established that the description of the three-dimensional system with finite number of particles $N$ near the critical point in the range of the values $\tau^{*}<\tau<\tau_{\mathrm{c}}$ and $h^{*}<h<h_{\mathrm{cr}}$ is valid only in the case of $N>N^{*}$, where

$$
N^{*} \approx\left[\left(h^{*}\right)^{2}+\left(\tilde{\tau}^{*}\right)^{5 \nu}\right]^{-3 / 5},
$$

where $\tilde{h}^{*}$ and $\tilde{\tau}^{*}$ are renormalized in accordance with the values of the field and temperature in the expression (5).

\title{
References
}

1. Stanley H. Introduction to Phase Transitions and Critical Phenomena. Clarendon Press, Oxford, 1971.

2. Ma S. Modern Theory of Critical Phenomena. Bengamin, Reading, (MA), 1976.

3. Belanger D.P. Braz. J. Phys., 2000, 30, 682.

4. Folk R., Holovach Yu., Yavors'kii T. Uspiekhi Fizichieskikh Nauk, 2003, 173, 175. [Physics-Uspiekhi, 2003, 46, 169].

5. Guida R., Zinn-Justin J. Nucl. Phys. B, 1977, 489, 626.

6. Zinn-Justin J. Quantum fiels theory and critical phenomena. Oxford Science Publications, Oxford, 1989.

7. Parisi G. J. Stat. Phys., 1980, 23, 49; Statistical field theory. Addosin-Wesley, Reading, MA, 1988.

8. Ferrenberg A.M., Landau D.P. Phys. Rev. B, 1991, 44, 50-81.

9. Guttman A.J., Enting I.G. J. Phys. A, 1994, 27, 8007.

10. Kozlovskii M.P. Phase Transition, 2007, 80, No. 1-2, 3-9.

11. Kozlovskii M.P. Condens. Matter Phys., 2005, 8, 473.

12. Kozlovskii M.P., Pylyuk I.V., Prytula O.O. Phys. Rev. B, 2006, 73, 1774406.

13. Yukhnovskii I.R., Kozlovskii M.P., Pylyuk I.V. Microscopic theory of phase transitions in the threedimensional systems. Eurosvit, Lviv, 2001, 592 p.

14. Kozlovskii M.P. Ferroelectrics, 2005, 317, 25.

15. Kadanoff L.P. Physics, 1966, 3, 263.

16. Engels J., Fromme L., Seniuch M. Nucl. Phys. B, 2003, 655, 277.

17. Kozlovskii M.P., Pylyuk I.V., Prytula O.O. Physica A, 2006, 369, 562.

\section{Вплив скінчених розмірів системи ізінгівських спінів на поведінку кореляційної довжини при наявності зовнішнього поля}

\author{
М.П.Козловський \\ Інститут фізики конденсованих систем НАН України, 79011 Львів, вул. Свєнціцького, 1
}

Отримано 4 травня 2007 р., в остаточному вигляді - 31 травня 2007 р.

\begin{abstract}
Отриманий явний аналітичний вираз для ефективної кореляційної довжини $\xi$ ізінгоподібної системи поблизу температури фазового переходу $T_{\mathrm{c}}$ як функції відносної температури $\tau$, зовнішнього поля $h$ та розмірів системи $L$. З'ясована роль цих величин у формуванні значення кореляційної довжини $\xi$. Показано, що аномальний ріст кореляційної довжини має місце лише у випадку $\tau \rightarrow 0, h \rightarrow 0$ та $L \rightarrow \infty$. При відхиленні від цих значень має місце суттєве сповільнення росту $\xi$. Для фіксованого розміру системи встановлений критерій допустимого діапазону температур (полів), де поведінка кореляційної довжини визначається температурною (чи польовою) змінною. Поза цим діапазоном $\tau<\tau_{\mathrm{c}}, h<h_{\mathrm{cr}}$ розмір системи стає визначальним при формуванні кореляційної довжини.
\end{abstract}

Ключові слова: фазовий перехід, кореляційна довжина, зовнішнє поле, скінченна система

PACS: $05.50 .+q, 64.60 . F r, 75.10 . H k$ 